\title{
Sport injuries in the paediatric and adolescent patient: a growing problem
}

\author{
Steven P. Kerssemakers • Anastasia N. Fotiadou • \\ Milko C. de Jonge • Apostolos H. Karantanas • \\ Mario Maas
}

Received: 21 October 2008 /Revised: 12 January 2009 / Accepted: 28 January 2009/Published online: 11 March 2009

C) The Author(s) 2009. This article is published with open access at Springerlink.com

\begin{abstract}
With an increasing number of paediatric and adolescent athletes presenting with injuries due to overuse, a greater demand is put on clinicians and radiologists to assess the specific type of injury. Repetitive forces applied to the immature skeleton cause a different type of injury than those seen in adults due to the differences in vulnerability of the musculoskeletal system, especially at the site of the growth cartilage. Intrinsic and extrinsic risk factors all play a role in the development of overuse injuries. MRI plays a key role in imaging overuse injuries due to its high potential for depicting cartilaginous and softtissue structures. Sport-specific biomechanics are described, since this knowledge is essential for adequate MRI assessment. An overview of several sport-related injuries is presented, based on anatomical location.
\end{abstract}

S. P. Kerssemakers

Department of Radiology, Medical Center Alkmaar,

Alkmaar, The Netherlands

S. P. Kerssemakers

Department of Radiology, Diakonessen Ziekenhuis, Leiden, The Netherlands

\author{
A. N. Fotiadou \\ Department of Radiology, "Papageorgiou" General Hospital, \\ Thessaloniki, Greece
}

M. C. de Jonge $\cdot$ M. Maas $(\bowtie)$

Department of Radiology, Academic Medical Center,

University of Amsterdam,

Meibergdreef 9, suite G1-211,

Amsterdam 1105, AZ, The Netherlands

e-mail:m.maas@amc.uva.nl

\author{
A. H. Karantanas \\ Department of Radiology, University of Crete, \\ Heraklion, Greece
}

Keywords Sports imaging $\cdot$ MRI $\cdot$ Trauma $\cdot$ Children

\section{Introduction}

In Western societies sport injuries in the paediatric and adolescent population are a clinical entity that is being seen more frequently [1]. Acute sport injuries are often easily recognized as there is a sudden onset of symptoms following a traumatic event, such as a direct force applied to the bones or joints. This can lead to fractures, in which the growth plate can be involved, or dislocations that often can be recognized clinically. There is appropriate awareness and the pattern of injury is known to paediatric and general radiologists. Acute soft-tissue injuries, tendon ruptures or ligamentous injury, are not so familiar and may therefore be more difficult to diagnose.

This paper focuses on overuse injuries, aiming to increase awareness amongst radiologists. Overuse injuries are caused by repetitive microtrauma with biomechanics that are sport-specific. They can lead to functional impairment and if not accurately appreciated and treated can even cause permanent disability. When a minor or trivial trauma causes disproportionate symptoms in an athlete, an overuse type of injury should be ruled out.

Special consideration has to be made for unique injuries occurring in the growing skeleton [2]. Several contributing risk factors have been described [3]. These can be divided into intrinsic factors, such as the increased vulnerability to stress in the growing skeleton and inability to detect injuries, and extrinsic factors, which include the growing pressure from peers, parents and trainers to perform better; also the lack of training and competition programmes adapted to the paediatric and adolescent population can play a role. Especially in the adolescent population, where 
there is an increasing tendency towards a professional level of sport activity, the incidence of overuse injuries is increasing rapidly [3]. Furthermore, there is an increased awareness amongst doctors of these types of pathology, which leads to a perceived increase in the problem.

For the general and paediatric radiologist who will probably be increasingly confronted with imaging of sport injuries it is important to understand these risk factors. In order to apprehend the types of injury it is important to understand the different biomechanical forces applied to joints and surrounding structures during various sports activities. In this review both clinical and imaging findings in the most common overuse injuries of the upper and lower limbs and spine of the immature athlete are presented with an emphasis on MRI.

\section{Intrinsic risk factors}

Bone growth is initiated at the site of the physis and epiphysis. There are two types of epiphysis in the extremities: pressure and traction epiphyses (apophyses). Pressure epiphyses are found at the proximal and distal end of the longitudinal bones. Longitudinal bone growth takes place from the growth plate (physis) between the metaphysis and epiphysis; therefore injuries to the epiphysis and/or physis can result in longitudinal bone growth disturbance [4]. Growth cartilage appears to be more vulnerable to stress than adjacent bone structures and adult cartilage, especially during periods of rapid growth [4, 5]. In children repetitive forces applied to long bones will more likely result in injury to the weaker epiphyseal plate than ligament disruption [5].

Traction epiphyses (or apophyses) are found at the insertion site of muscles and tendons and play a role in the development of bone shape but not in longitudinal bone growth. As a skeletal muscle and its tendon are more resistant to stress than the apophyseal growth plate, repetitive microtrauma can lead to traction apophysitis, which in turn can lead to an apophyseal avulsion $[2,5]$.

The growth of the musculotendinous units is secondary to longitudinal bone growth. During growth spurts the lagging muscle and tendon growth can create tightness and inflexibility around joints whilst during sport activity increased stress is applied to both joints and musculotendinous structures. Imbalance can also occur due to uneven flexibility and strength of the surrounding muscle groups. Preexisting misalignments increase the likelihood of such imbalances.

Physiological factors include decreased bone density in amenorrhoeic female athletes, skeletal maturity, muscle bulk of the individual and deconditioning and poor preparation. Finally, psychological factors such as high- risk behaviour in adolescents can play a role in developing sport injuries [5].

\section{Extrinsic risk factors}

The most important extrinsic risk factor is inappropriate training. Training programmes ideally are adjusted to skeletal maturity, decreased flexibility of muscles and tendons and level of condition in every individual. Coaches and trainers should pay extra attention to technique and excessive forces applied to the immature body. Sporting equipment should ideally be optimized for the less wellprepared participant. Attention should be given to the environment, especially the surface on which the activity is to be performed $[3,5]$. Another extrinsic risk factor in this young athletic population is thought to be parental pressure [6].

\section{The upper limb}

Overuse injuries to the upper limb are mostly found in throwing athletes and gymnasts who exert great pressure forces on their arms.

The basic motion in all throwing sports is identical. It is divided into five stages [7]:

1. Wind-up with minimal stress

2. Early cocking with abduction and external rotation of the shoulder

3. Late cocking with great shear forces applied to the glenohumeral joint mainly due to rotator cuff muscle activity

4. Acceleration with tremendous stress on the elbow joint, with at the same time exertion of great compression and rotational forces on the radiocapitellar joint and traction at the posterior compartment

5. Follow-through characterized by rapid deceleration with muscle activity aimed at glenohumeral stability and prevention of anterior luxation

The shoulder

\section{Osseous and growth cartilage injuries}

A well-known overuse injury in throwing athletes in the physis of the proximal humerus leading is little leaguer's shoulder. Due to repetitive microtears widening of the physis occurs, shown on conventional radiographs; other findings include demineralization, sclerosis, fragmentation of the lateral aspect of the proximal metaphysis and cystic changes [8]. Sometimes additional radiographs of the 
contralateral side are necessary. When radiographs are negative, especially in an early phase, MRI can be helpful. Besides widening of the physis there is high-intensity signal in the metaphysis on T2-weighted (T2-W) images and hypointensity on the T1-weighted (T1-W) series representing marrow oedema $[9,10]$. Periphyseal oedema and subtle periosteal elevation and oedema have also been described [11].

\section{Intraarticular injuries}

Glenohumeral dislocation is relatively uncommon in the paediatric population; however it is the most common site for acute articular dislocation in adolescents. Plain radiographs in AP, axillary and lateral directions should be obtained as fractures of the glenoid (Bankart lesions) and/or posterior impression fractures of the humeral head (HillSachs lesions) can occur. Labral tears and capsular laxity have been described in throwing athletes, and MR arthrography is advised to confirm the diagnosis and plan the surgical repair $[7,12]$.

\section{Soft-tissue injuries}

Cuff pathology is relatively uncommon in the paediatric and adolescent population, as the epiphyses (at this site the humeral tubercle) is the weakest link of the musculoskeletal chain. MRI is the imaging modality of choice to depict pathology [7, 13].

The elbow

\section{Osseous and growth cartilage injuries}

In immature athletes, most chronic elbow injuries are sustained due to repetitive valgus stress often associated with throwing activities or gymnastics. As some authors place chronic elbow pain of all causes in young throwing athletes under this denominator, little leaguer elbow is regarded as a traction apophysitis of the medial epicondyle mostly occurring in the 9-14-year-old age group due to repetitive valgus stress [14]. Radiographic examination can be completely normal or show subtle widening of the physis or fragmentation of the apophysis, although these findings are also described in athletes without complaints [15]. Often MRI is necessary to confirm the diagnosis and exclude differentials. On proton density/T2-W images with fat saturation there is often bone marrow oedema in the medial epicondyle together with increased signal in the surrounding soft tissue and the flexor tendons (Fig. 1). Due to the valgus stress with traction at the medial side but compression forces at the lateral side, osteochondral changes in the capitellum and premature closure of the radial epiphysis can occur [16] (Fig. 1). Traction apophysitis of the olecranon is a relatively rare condition, caused by repetitive forceful contractions of the triceps muscle. Patients present with a dull pain with tenderness over the olecranon. It is characterized by widening of the epiphyseal plate and fragmentation of the apophysis. In adolescents, with a more mature apophysis the same mechanism can cause a transverse fracture through the growth plate $[14,16]$.

\section{Intraarticular lesions}

An osteochondral lesion, also known as osteochondritis dissecans, typically occurs in children and is mostly located in the capitellum or trochlea. Due to repetitive valgus stress or axial loading, especially in pitchers and gymnasts, subchondral infarction occurs, followed by chondral lesions and dehiscence of bony fragments (Fig. 2). A variant of osteochondral lesion is Panner's disease, which can be considered a self-limiting osteochondrosis in the under10-years age group due to avascularity of the capitellum rather than a posttraumatic disorder [7, 17]. MRI findings include high signal intensity of the subcortical bone on T2-W images, fragmentation with defects in both subchondral bone and overlying cartilage and loose fragments, which can sometimes best be depicted on CT arthrography if MRI is inconclusive.

\section{Soft-tissue injuries}

Lateral epicondylitis (tennis elbow) and medial epicondylitis (golfer's elbow) are stress reactions at the insertion sites of extensor and flexor muscle tendons due to longstanding repetitive overuse. As the apophysis forms the weakest point in the musculoskeletal chain this type of injury is rare; imaging is seldom necessary and serves mostly to exclude other lesions. Ulnar collateral ligament ruptures are rare in children and adolescents as the ligament is stronger than the attached structures and generally occur after epiphyseal fusion [18].

\section{Wrist/hand}

\section{Osseous and growth cartilage injuries}

Scaphoid fractures in adults are mainly located in the waist, whereas in children it is mostly the distal part that is fractured; almost half of these scaphoid fractures are avulsions. In scaphoid fractures MRI has replaced bone scans, due to its superior specificity. However, when there is only a cortical avulsion injury, bone marrow oedema is not always that striking. A typical overuse injury is gymnast wrist (Fig. 3) caused by a combination of 

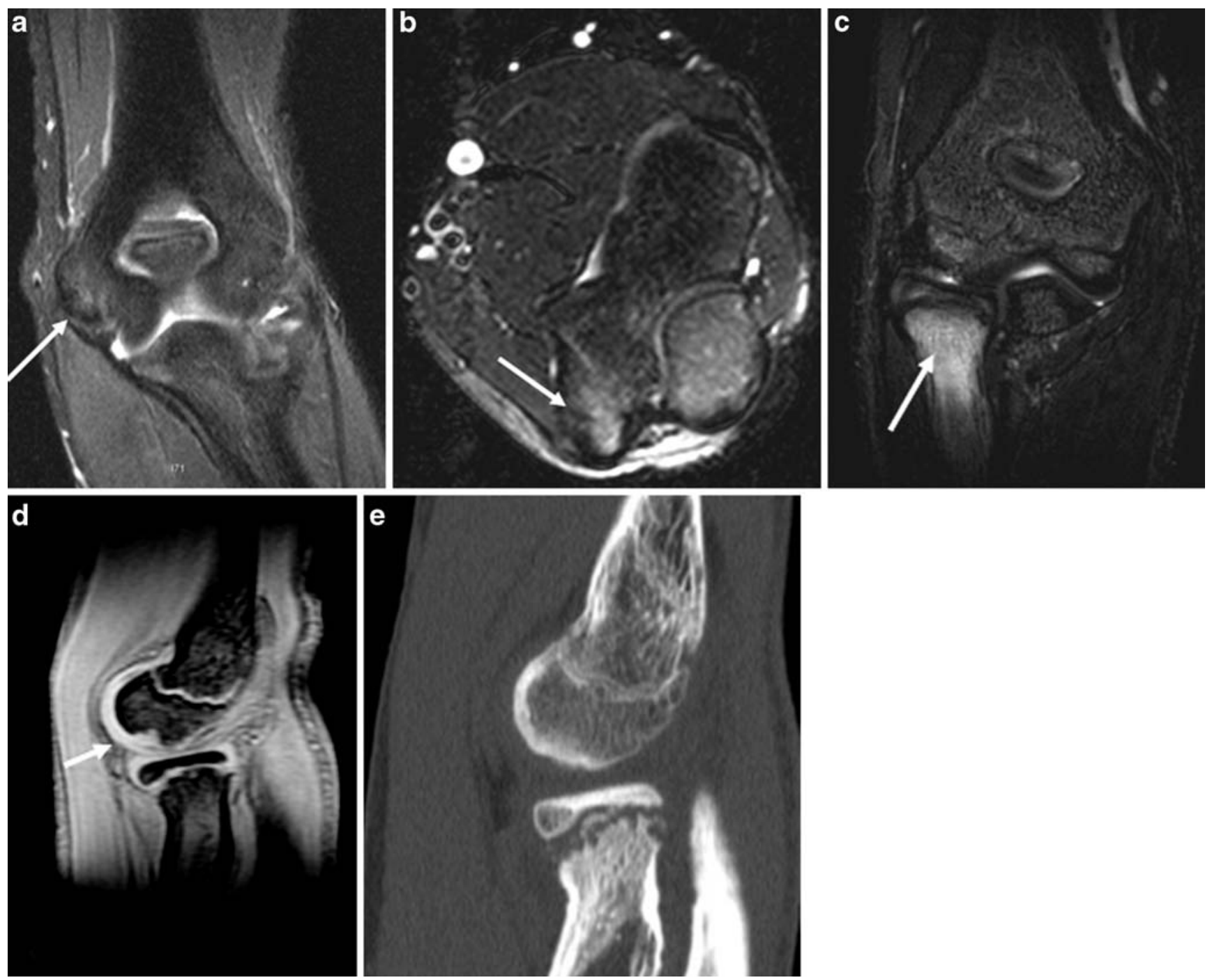

Fig. 1 Valgus stress injuries. a, b A 15-year-old elite-level gymnast presented with chronic medial elbow pain. Coronal (a) and axial (b) T2-W fat-saturated MR images show oedema in the medial epicondyle representing the traction apophysitis at this site (arrow). c-e An 11-year-old gymnast complained of medial elbow pain after repetitive exercise with compression forces at the lateral elbow compartment.
Coronal (c) and sagittal (d) MR images show marrow oedema in the proximal radial metaphysis (arrow) as well as irregular aspect of the physis and epiphysis. There is also oedema in the capitellum surrounding a small osteochondral lesion. e CT image shows marked irregularity at the site of physis and epiphysis with fragmentation representing chronic epiphyseal plate injury repetitive axial loading and hyperextension of the wrist; physeal microtears lead to growth disturbance of the distal radius, ulnar plus variant and subsequent chondral damage. Radiographs can show the ulnar plus deformation as well as widening and irregularity of the physis, narrowing of the epiphysis and sclerosis or cystic changes in the metaphysis [19]. If inconclusive, MRI can depict physeal cartilage extension into the metaphysis, bone bruise or vertical fractures [20].

In tennis, stress-related injury with bone marrow oedema in the carpal bones is recognised.

In adolescent rock climbers presenting with chronic finger pain and decreased motion, stress reactions such as cortical hypertrophy and subchondral sclerosis, as well as stress fractures of the proximal physeal plate (Salter-Harris type II or III) of the middle phalanx (mostly of digits 3 or 4) have been described, and lead to osteoarthritis [21, 22].

\section{Intraarticular and soft-tissue injuries}

Ligamentous or tendon ruptures are rare. In patients with persisting complaints, MRI can be useful in detecting injuries such as triangular fibrocartilage complex (TFC) tears or intercarpal ligament disruption [12] (Fig. 4). The extensor carpi ulnaris tendon can show subluxation from its groove with a lesion of the retinaculum, especially in tennis 

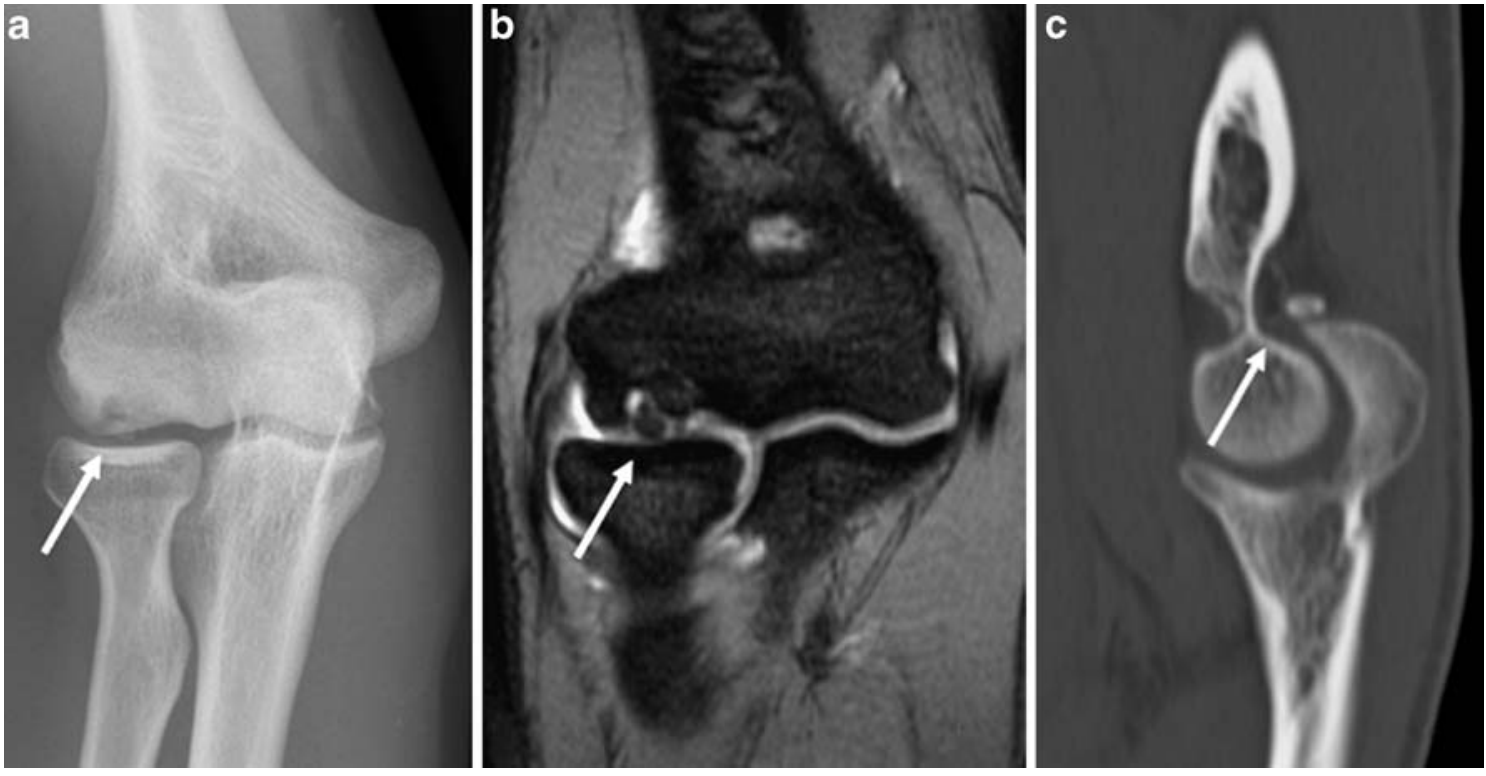

Fig. 2 Osteochondral lesion of the capitellum in a 16-year-old gymnast with chronic elbow pain. a Plain radiographs show the osteochondral lesion of the capitellum with a loose body (arrow). b Coronal 3-D gradient-echo MRI arthrography depicts the loose

as a result of a stressed back hand. Ultrasonography is superior to MRI in depicting this dynamic pathology.

\section{The spine}

Chronic low back pain is a common complaint in the paediatric and adolescent athlete. Especially athletes such as gymnasts and wrestlers, who put excessive axial loading as well as flexion and extension forces on their spine, are at risk [23].

Spondylolysis is a common finding among young athletes and can occur with or without spondylolisthesis. Spondylolysis is, however, a common finding in the whole population with a prevalence of $6-8 \%$. Thus the exact role of this finding remains an issue of discussion. There are several classification systems for spondylolysis. For children and adolescents the scheme can be as follows: (a) dysplastic, (b) developmental, (c) traumatic and (d) pathological [24]. In the dysplastic type there is an intact pars interarticularis. In the developmental type there is a bony gap and surrounding sclerosis; in this group heredity has been demonstrated. The traumatic type can be divided into an acute and chronic form. In the chronic traumatic form there are three subtypes: stress reaction with only oedema on MRI without a bony gap; a stress fracture in which there is oedema and cortical disruption without a large bony gap; and finally, spondylolysis with a larger bony defect with sclerotic changes on both sides of the pars interarticularis. fragment as well as the cartilage injury to both radial head and capitellum (arrow). c Sagittal reformatted CT image in another 16-year-old patient shows a loose body in the fossa olecrani (arrow), not noted on conventional radiographs or MRI

Conventional radiography will often be negative and oblique views are not advised due to lack of sensitivity and radiation burden. MRI is the imaging modality of choice, depicting bone marrow oedema, signal changes in adjacent muscles, and disc degeneration secondary to spondylolisthesis without the use of ionizing radiation, especially in an early stage [25-27]. Helical CT with multiplanar reconstruction (MPR) better detects the extent of the lesion (Fig. 5). However, it remains unclear whether this staging is

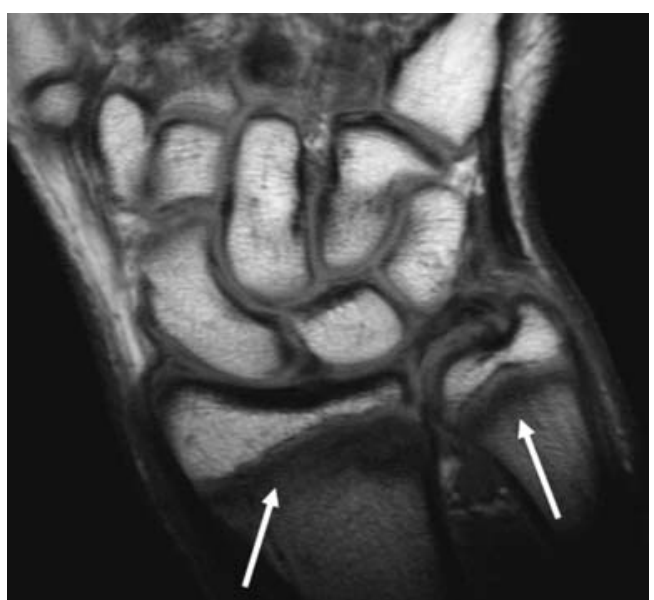

Fig. 3 Gymnast wrist. A 12-year-old gymnast with chronic wrist pain. Coronal T1-W MR image shows marked broadening of the irregular distal radial and ulnar physis. There is an oedematous zone with low signal extending into the distal metaphysis (arrows) 

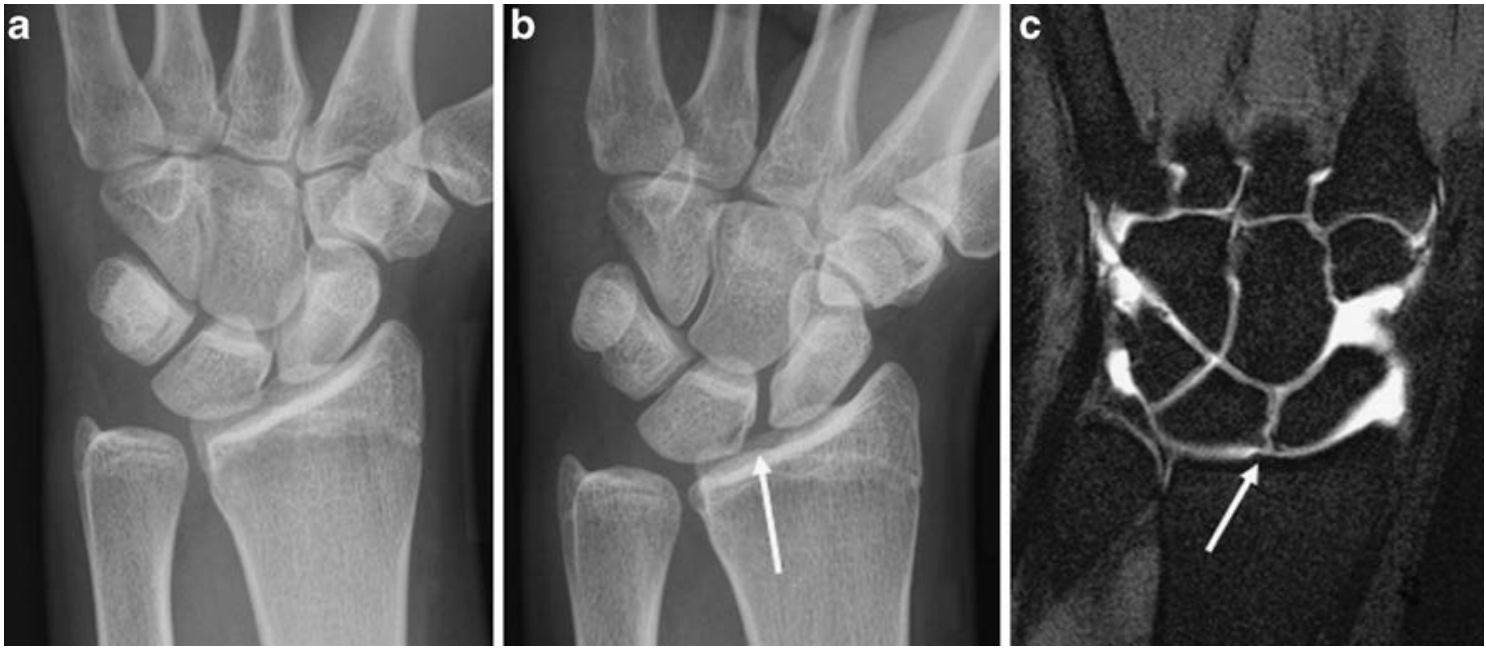

Fig. 4 Scapholunate ligament injury. A 16-year-old judoka sustained a wrist injury and kept complaining of pain for several weeks. a Initial radiograph is negative. b Radiograph with clenched fist shows subtle

correlated with the clinical situation and it is not possible to offer prognosis in each individual case.

Spondylolisthesis can be graded on lateral/sagittal views as $\leq 50 \%$ slippage, which is considered stable, and $>50 \%$ slippage, which is unstable [28]. Vertebral deformation with wedging and disc protrusion/degeneration appears to be secondary to spondylolisthesis instead of being a contributing factor [29].
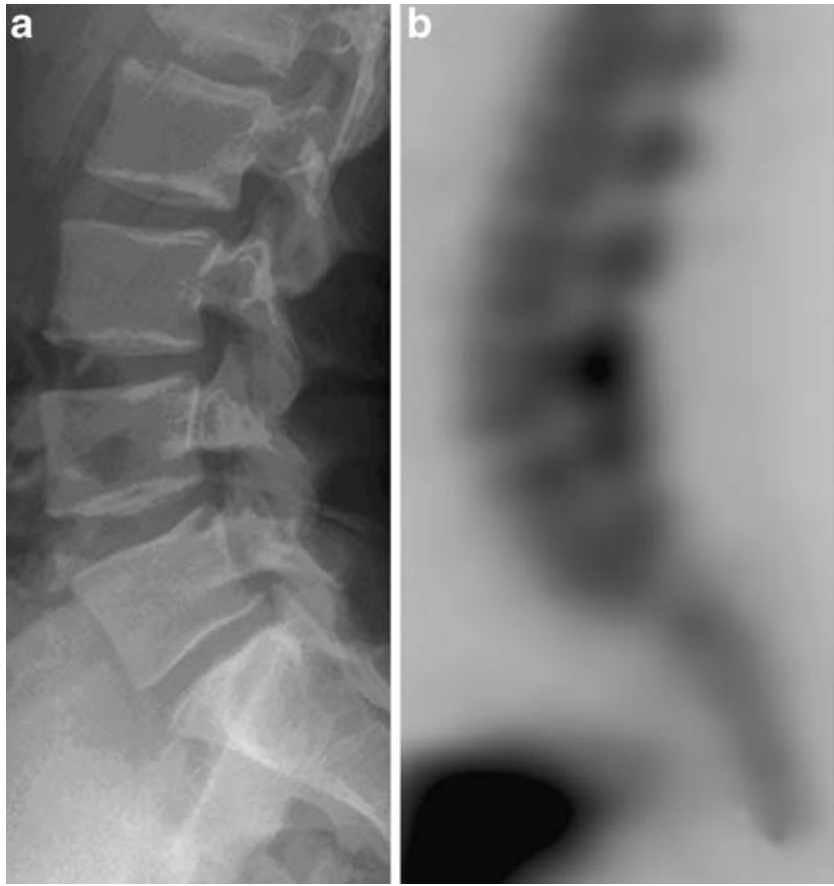

Fig. 5 Spondylolysis. A 15-year-old soccer player complained of persistent low back pain following a fall 4 months earlier. a Plain radiograph is negative. b SPECT image shows increased uptake at the level of the pedicle of L4. c On T2-W sagittal MR image there is widening of the space between the scaphoid and lunate (arrow). $\mathbf{c}$ MR arthrography depicts the scapholunate intercarpal ligament rupture (arrow)

Vertebral ring apophyseal injury occurs mainly in adolescent athletes, as the apophyseal ring appears to be most vulnerable in this age group that is characterized by rapid growth. Controversy exists whether the condition is the result of trauma, overload, or a growth disorder [23].

Anterior apophyseal injury can be the result of intravertebral disc herniation or due to traction by muscular activity or lumbar hyperextension. As cartilaginous structures
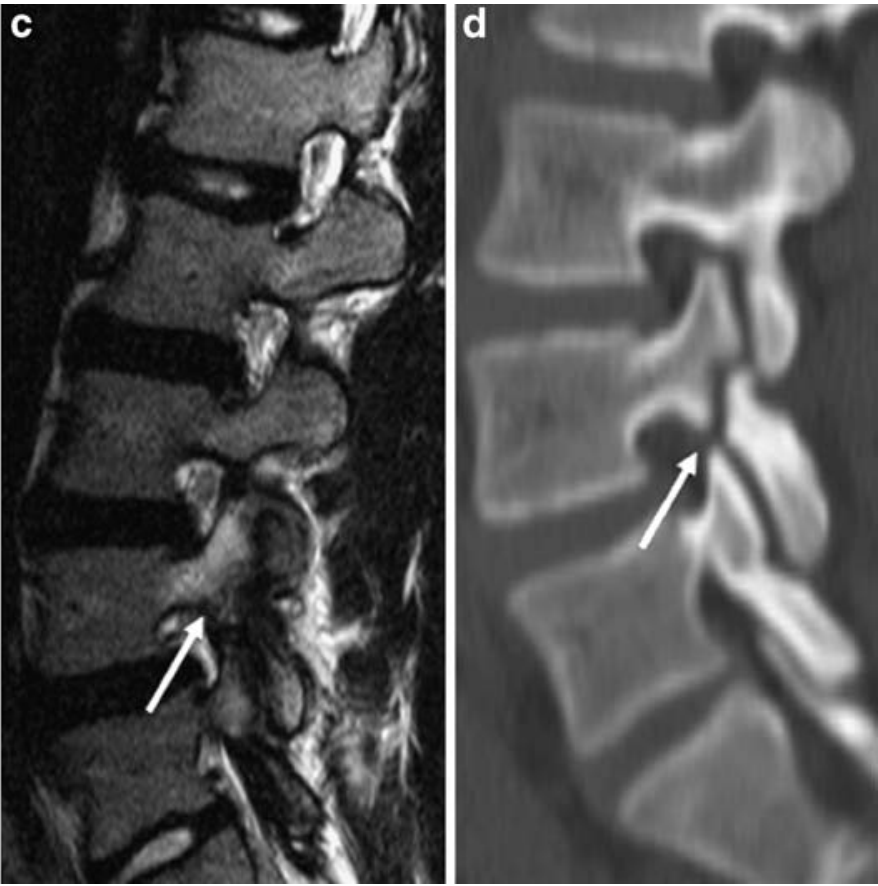

increased signal intensity in the pedicle of L4 (arrow) and suspicion of cortical disruption in the interarticular process of L4. d CT MPR image best shows the osseous defect in the pars interarticularis (arrow) 
are involved, MRI is considered the most sensitive modality [23].

Posterior ring apophyseal fracture is a rare condition in which neurological deficit can be the reason for surgical intervention. As radiographs can be negative and MRI appears to have low sensitivity, CT is the gold standard to show the fragment avulsed into the spinal canal [30].

Although the exact aetiology of the disease is unknown, the thoracolumbar form of Scheuermann disease is found more frequently in athletes than in non-athletes. Findings include disc degeneration, reduced disc height, Schmorl nodes and flattening of vertebral bodies with kyphotic deformation, all of which can be seen on plain radiographs [23].

\section{The lower limb}

Pelvis-hip-thigh

\section{Osseous injuries}

In the growing skeleton, a forceful contraction of an attached muscle may separate the apophysis leading to an avulsion fracture [31, 32]. Acute apophyseal avulsions are mostly noncontact injuries and typically present with severe and well-localized pain. Plain radiographs will confirm the presence of immature apophyses and clarify the degree of displacement (Fig. 6). Follow-up radiographs may show calcification filling the defect at the avulsion site. Solid callus formation is not necessary for full functional recovery. Small cortical avulsion fractures may not be visible on MRI [33].

Chronic or recurrent apophyseal injuries may result in irregular patterns of ossification around the pelvis that can easily be confused with other more aggressive disorders [34]. CT is the method of choice for assessing the benign origin of the lesion. High-intensity activity might be associated with repetitive traction on an apophysis without avulsion. MRI in these athletes who present with pain shows soft tissue and bone marrow oedema. Marrow oedema, demonstrated with high signal intensity on fatsuppressed images, represents a stress response of the cancellous bone due to microfractures of the trabeculae [35]. Stress fractures, located in the sacrum, acetabulum, and femoral neck, as well as pubic osteitis, which is a stress response, may occur in the growing skeleton [36].

\section{Intraarticular injuries}

The hip joint, with its deep-seated ball-in-socket morphology, is quite stable and protected. As a result, osteochondral injuries and labral tears are very rare. In sports that provoke repetitive maximized stress, a subchondral stress fracture may be depicted in the femoral head (Fig. 7). Plain radiographs and MRI are able to depict the lesion [37].
Fig. 6 Acute avulsion injury in a 15-year-old male football player at the insertion of the hamstrings. a The AP plain radiograph shows the avulsion of the ischial tuberosity (arrow). The bone fragment is well defined and displaced inferiorly. $\mathbf{b}$, c MDCT images in the axial (b) and coronal (c) planes show to better advantage the degree of displacement (arrows), which is less than $2 \mathrm{~cm}$. d, e STIR MR images in the transverse (d) and coronal (e) planes show the associated muscular strains in the adductor and hamstring muscles respectively (thick arrows). The avulsion site is also shown (thin long arrow)
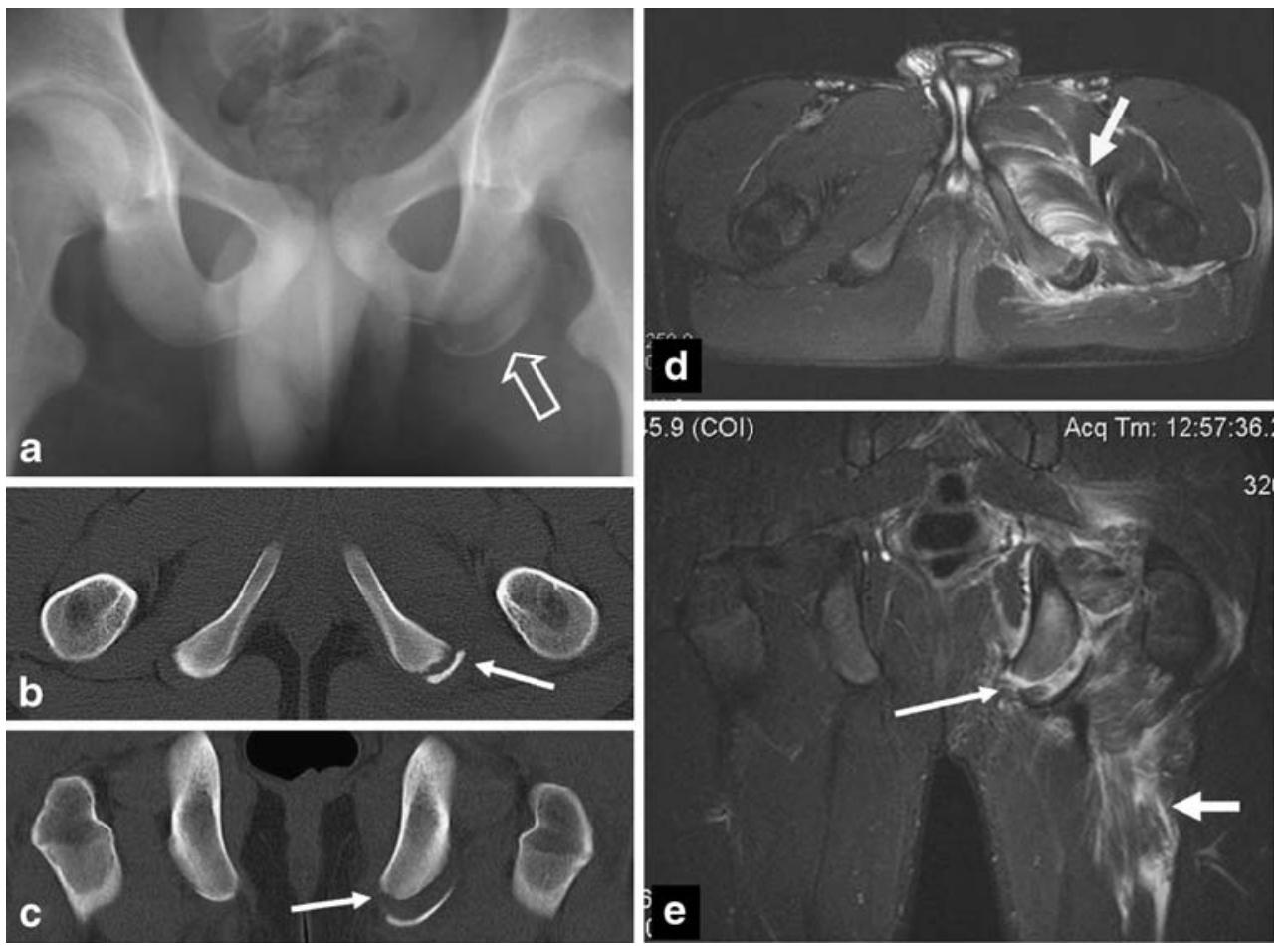

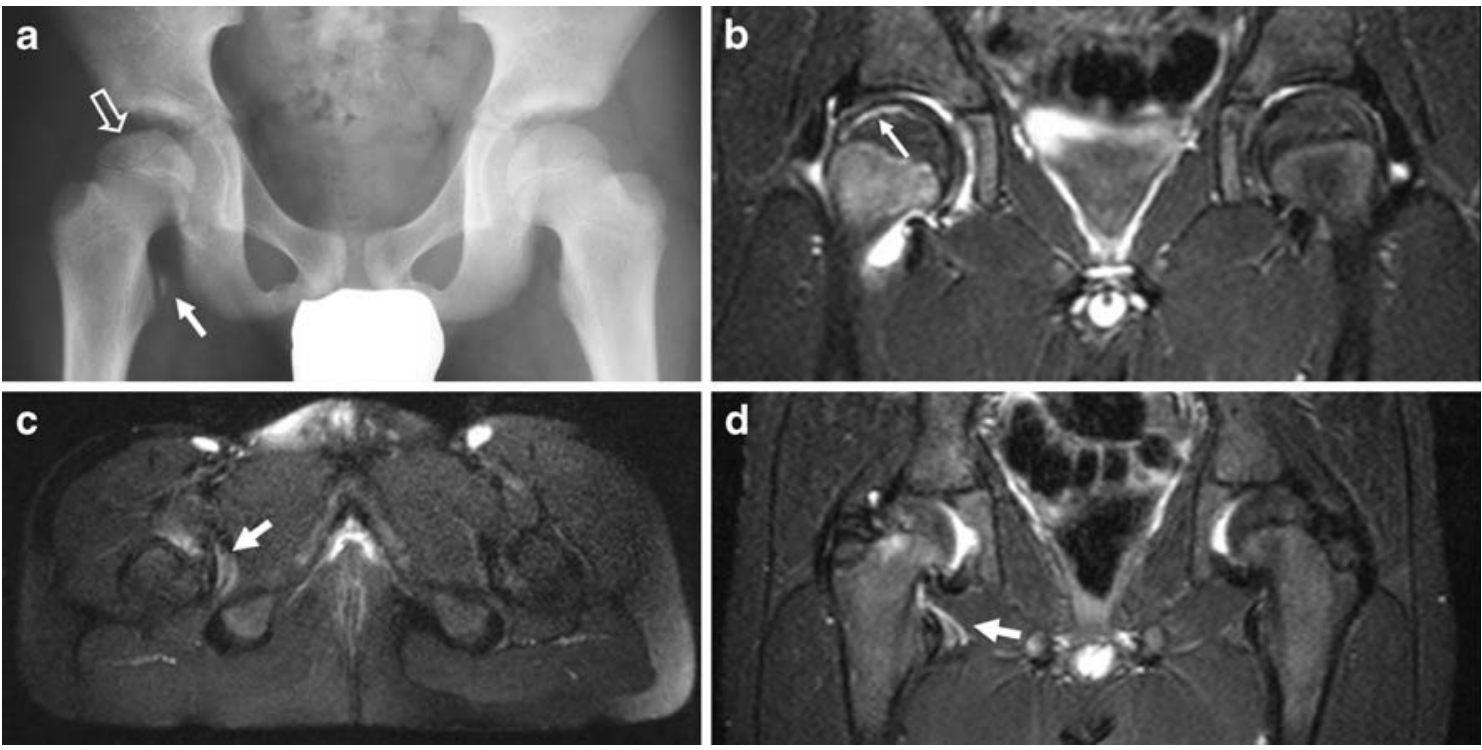

Fig. 7 Subchondral stress fracture in a 10-year-old male football player presenting with acute pain at the right medial thigh. There was also a history of an insidious right hip pain during the previous month. a The AP radiograph shows an avulsion fracture in the lesser trochanter (arrow). Localized flattening of the superolateral aspect of the femoral head with a subchondral lucency is also seen (open

\section{Soft-tissue injuries}

Muscle contusions result from direct blows. A lump may be palpable and MRI might be indicated to exclude a sarcoma by depicting the intramuscular oedema and haemorrhage.

Myotendinous strains are classified as grade I (stretch injury), grade II (partial tear), and grade III (complete rupture). MRI reveals high signal oedema on T2-W and STIR images (Fig. 6), as well as high-signal haemorrhage on T1-W images [38]. Acute tears usually occur in the hamstrings and quadriceps muscles. Dynamic US with active muscle contraction is an accurate test for diagnosing these injuries. Lateral hip pain over the greater trochanter may be due to trochanteric bursitis, apophysitis, or gluteus medius tendinopathy.

Thigh splints represent an overuse injury from continued traction of the vastus and adductor muscles to their attachment resulting in local periostitis. MRI reveals focal intramedullary oedema and periosteal reaction on the medial aspect of the femur [39].

\section{Knee}

\section{Osseous injuries}

Transverse fractures occur following direct blows, whereas spiral fractures are common in twisting injuries, especially in contact sports and mountain skiing. Transphyseal Salter- arrow). b Coronal STIR MR image shows the high signal intensity subchondral meniscal lesion in keeping with a fatigue subchondral fracture (arrow). c, d STIR MR images in the axial (c) and coronal (d) planes at the level of the avulsion injury show the oedema at the insertion of the iliopsoas tendon (arrows), but not the bone fragment itself

Harris fractures are also frequent. Occult on plain radiographs, tibial or femoral fractures can be depicted by MRI [40] (Fig. 8).

In the child, the physeal plate is weaker than the ligaments. Therefore, valgus and varus stresses result in injuries to the growth plate rather than the collateral ligaments. Premature epiphyseal fusion is a complication, diagnosed with both plain radiographs and MRI [34] (Fig. 9).

Overuse injuries are more commonly encountered in running sports. The continued traction of the muscles to their attachment causes local periostitis, mainly in the anterior tibia (shin splints). MRI shows periosteal oedema with or without cortical oedema [41]. Stress fractures are usually seen in the tibia [42]. Symptoms are typically insidious, but occasionally can be acute. Stress fractures occur especially in runners and in female athletes with osteoporosis, amenorrhoea and eating disorders. Radiographs obtained after injury or even at follow-up may appear normal. MRI is recommended as it is able to reveal bone marrow oedema as an early finding or the fracture as a low-signal intensity line on all sequences [43] (Fig. 10). Although scintigraphy is very sensitive, it is not specific and there is a high radiation dose of $2.3 \mathrm{mSv}$ (approximately 350 chest radiographs), so it is not advised.

Chondromalacia is the commonest cause of anterior knee pain in young athletes due to softening of the patellar articular cartilage. Patients usually have a history of pain associated with intense athletic activity requiring repetitive 

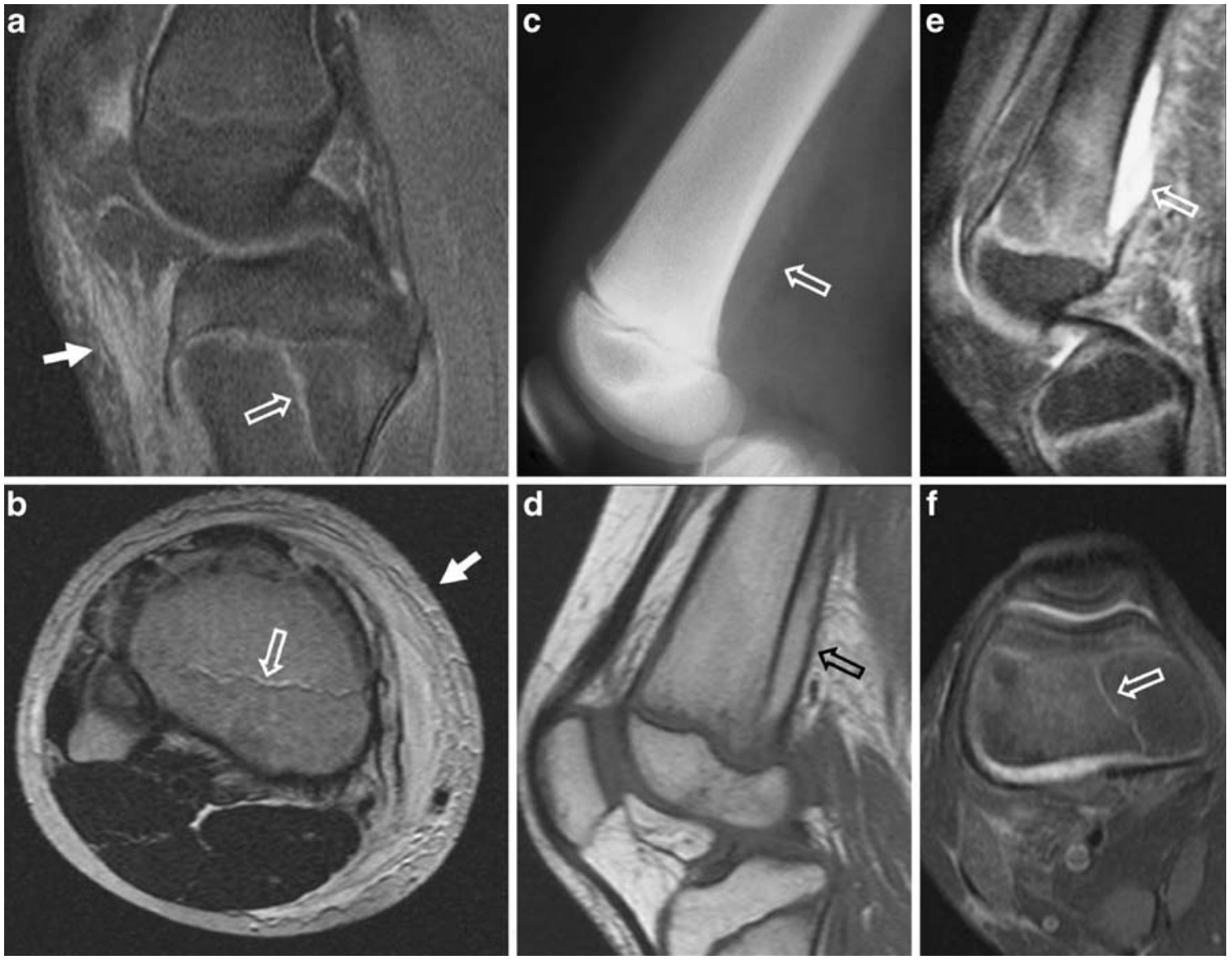

Fig. 8 Occult fractures. a, b A 15-year-old male football player who sustained an injury 2 days before imaging. The sagittal fat-suppressed proton density MR image (a) and the axial T2-W MR image (b) show the high-intensity signal from the fracture in the tibial metaphysis (open arrows). There is also soft-tissue haematoma anteriorly and medially secondary to the direct blow (white arrows). c-f A 9-year-old

female skiing athlete after a twisting injury. The plain radiograph (c) shows a density posterior to the distal femoral metaphysis suggesting haematoma formation (arrow). The corresponding sagittal T1-W (d) and fat-suppressed proton density (e) MR images show a subperiosteal haematoma (arrows). The axial fat-suppressed proton density MR image (f) shows an occult fracture (arrow)

Fig. 9 Growth arrest. An 11-year-old female skiing athlete with a previous epiphyseal injury. The plain radiograph (a) and coronal T1-W MR image (b) show growth plate fusion on the medial side (arrows)
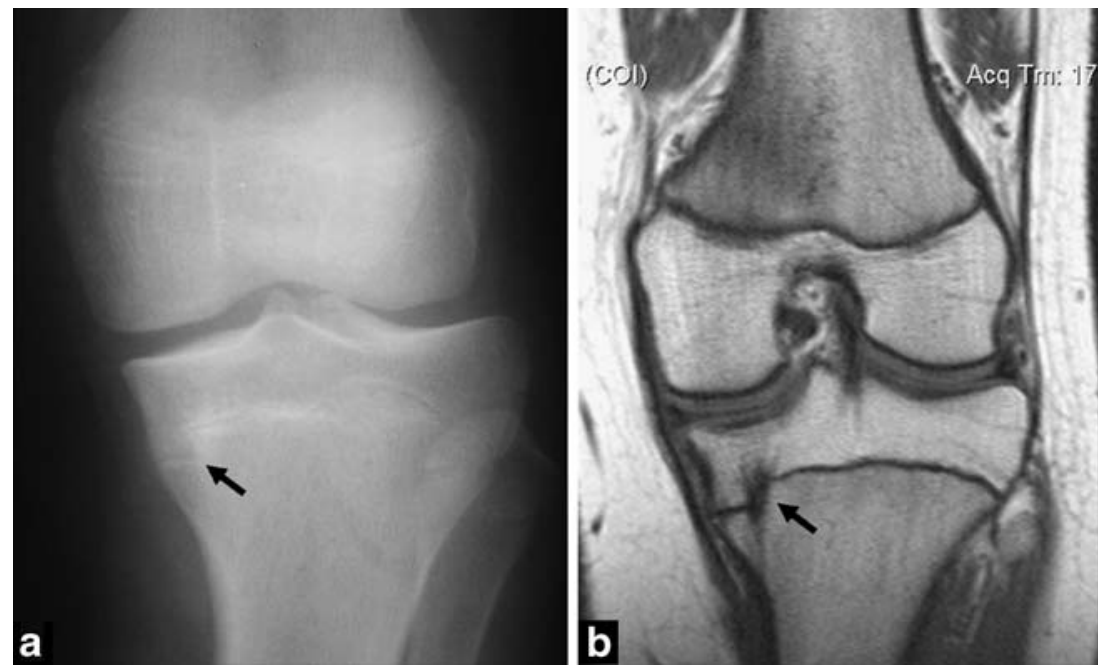
Fig. 10 Stress fractures. a, b A 15-year-old male footballer. Axial T1-W (a) and contrastenhanced T1-W (b) MR images show a periosteal reaction (arrows) in the fibula, with bone marrow oedema that enhances after contrast medium administration. c-e A 14-year-old male track athlete presenting with a 2-week history of proximal tibial pain, the coronal STIR MR image (c) shows extensive bone marrow oedema (arrows). The sagittal T1-W MR image (d) shows periosteal thickening (arrow). The plain radiograph (e) obtained 5 weeks after the onset of symptoms shows the periosteal thickening (open arrow) and trabecular sclerosis (white arrow) representing the stress fracture
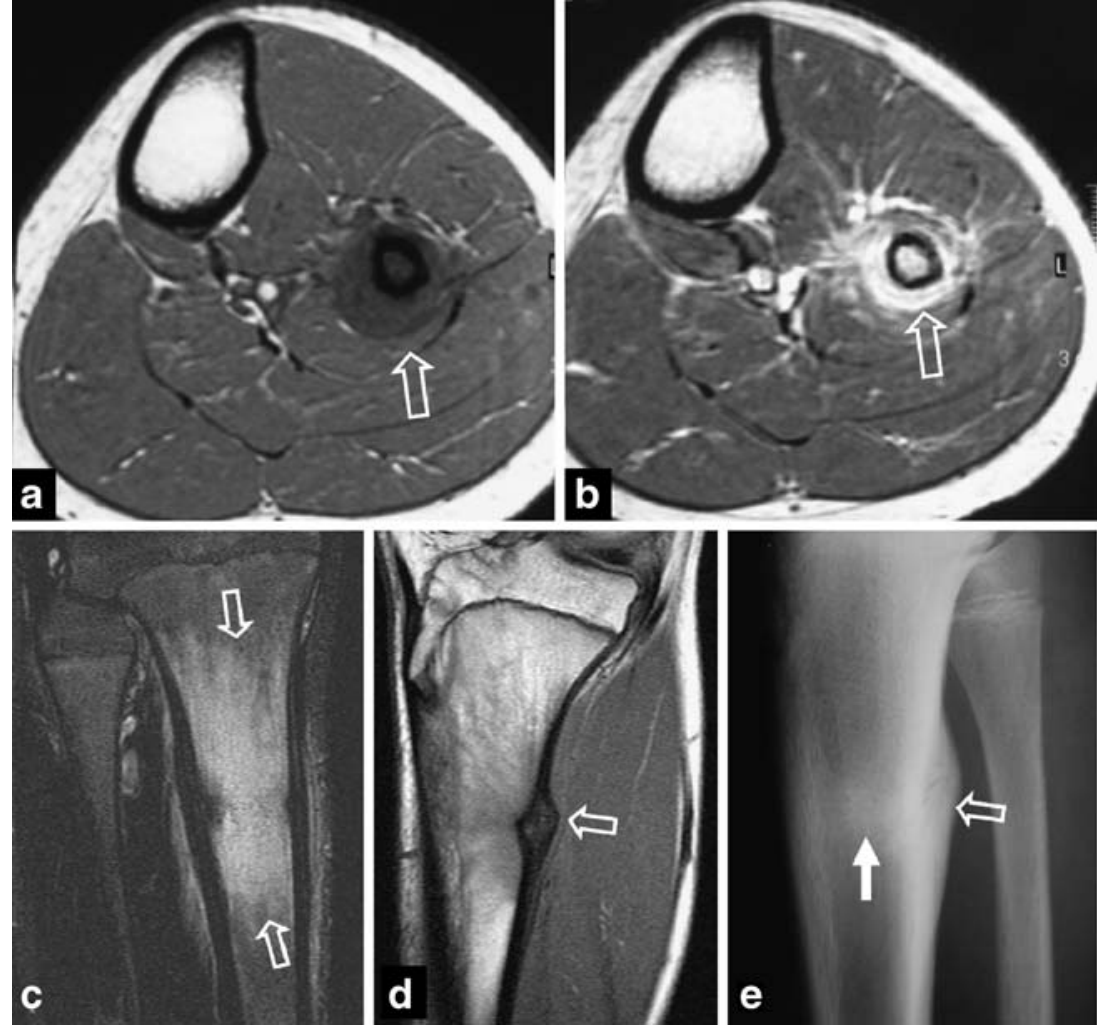

Fig. 11 Avulsion of the tibial eminence. a, b A 14-year-old male football player who had sustained a knee injury 3 days before imaging. Coronal T1-W MR image (a) shows the avulsion with minimal displacement (arrow). The axial fatsuppressed T2-W MR image (b) in the same patient shows a haemarthrosis and a droplet of fat in the effusion (arrow). c, d A 15-year-old male basketball player. The coronal fatsuppressed STIR MR image (c) shows a bone bruise in the lateral femoral condyle secondary to a patellar dislocation (arrow). The coronal T1-W MR image (d) 9 months after the dislocation shows avulsion of the tibial eminence (arrow)
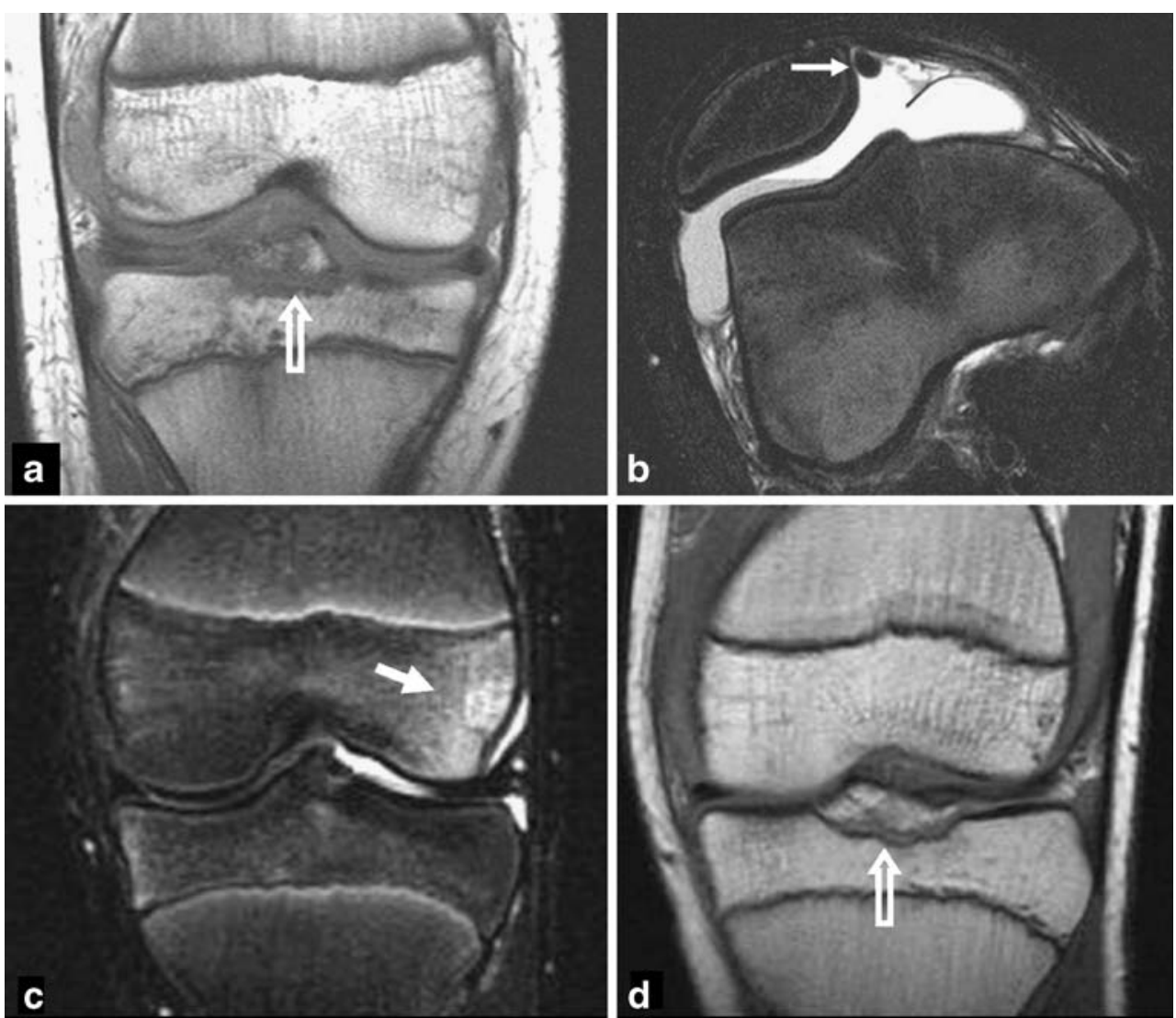
Fig. 12 Meniscal injury. A 14-year-old skier who was injured during a slalom 2 days before imaging. a The sagittal fat-suppressed proton density MR image shows an impacted osteochondral fracture in the medial femoral condyle (open arrow), the associated bone bruise (thin arrow) and the prepatellar haematoma (thick arrow). b The sagittal fatsuppressed proton density MR image shows a contusion of the lateral discoid meniscus (arrows) and a joint effusion
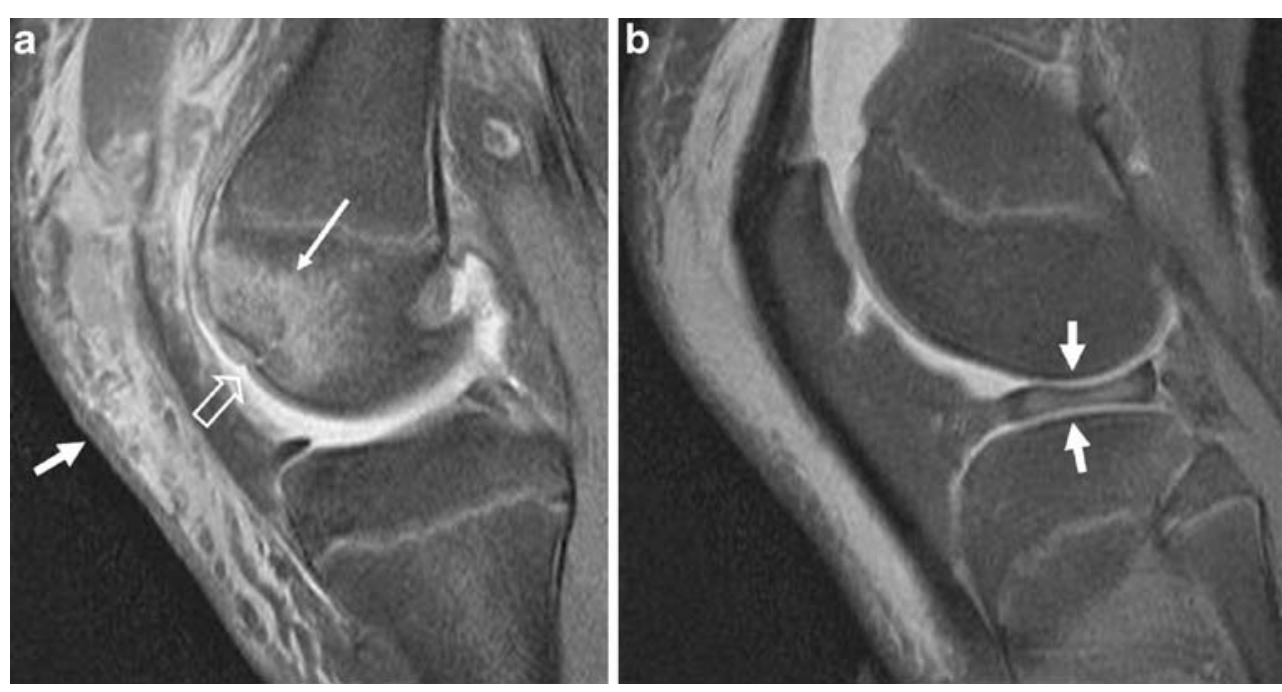

knee flexion. In the later stages plain films may show an illdefined posterior border of the patella or osseous defects and sclerosis. Radiographs may also reveal coexistence of a bipartite patella. MRI is able to depict early the extent of swelling or thinning of the patellar cartilage [44].

Sinding-Larsen-Johansson syndrome is a self-limiting condition with calcified tendinopathy in an avulsed distal apophysis of the patellar apex. Osgood-Schlatter syndrome is the corresponding tendinopathy on the ossification centre of the tibial tubercle by the distal patellar tendon. Radiographs in both syndromes show fragmentation of the lower pole of the patella and the tibial tubercle, respectively. MRI usually is not indicated.

\section{Intraarticular injuries}

A flexion, twisting, or hyperextension injury with immediate pain and haemarthrosis should raise the suspicion of anterior cruciate ligament (ACL) injury. ACL tears are rare in children but they often occur in adolescents. In skeletally immature patients a lateral radiograph may show avulsion of the tibial eminence. MRI can be performed to depict nondisplaced avulsions, to establish whether an ACL tear has indeed occurred, and to demonstrate associated abnormalities in the joint [45, 46] (Fig. 11).

Meniscal problems in this age group are unusual and are generally associated with a discoid lateral meniscus. Meniscal tears are most commonly seen in running, jumping, and pivoting sports. The medial meniscus is torn more commonly than the lateral meniscus. Most meniscal tears involve the posterior portion of the meniscus, resulting in tenderness over the less easily palpated posterior joint line. Meniscal contusion with a reversible swelling and oedema may occur. MRI is the modality of choice for diagnosing the injured meniscus [47] (Fig. 12).
Chondral and osteochondral injuries are significantly more prevalent than meniscal or ligamentous injuries in young athletes. Femoral lesions are more frequently encountered in children and patellar lesions in adolescents. MRI findings include cartilaginous thickening in grade I lesions, superficial ulceration or fissuring in grade II, deep ulceration or fissuring in grade III, associated bone bruise in grade IV and separated fractures in grade V. Impaction fractures may also occur (Fig. 12).

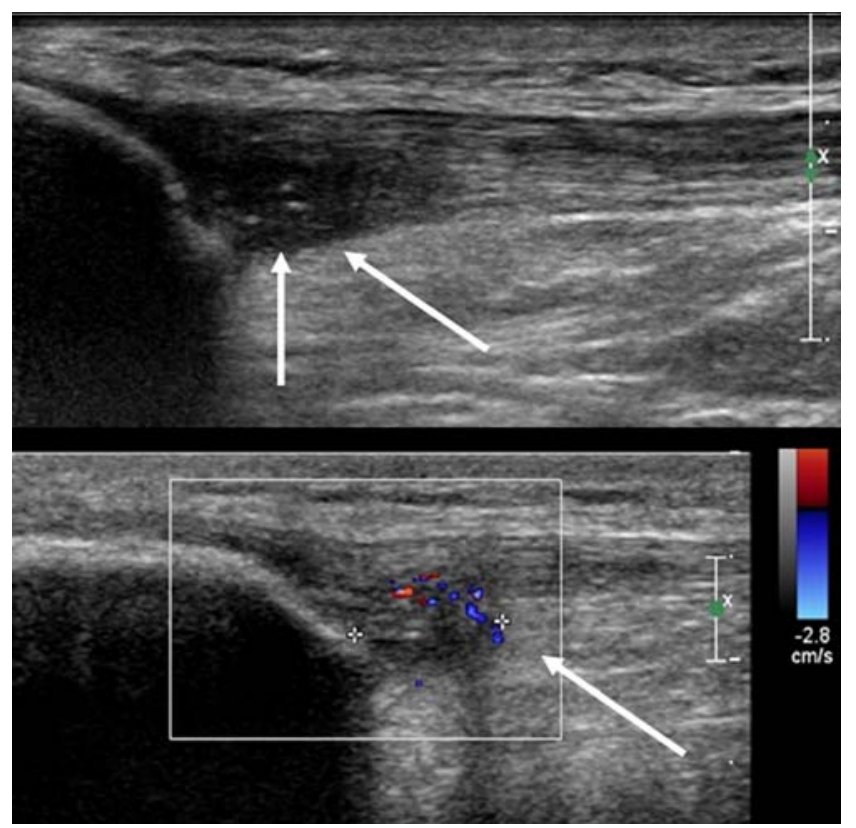

Fig. 13 Overuse injury, "jumper's knee" in a young professional tennis player. a Sagittal US image shows a broadened irregular proximal patellar tendon with small calcifications (white arrows). b Colour Doppler US image of this area shows neovascularization 
Osteochondritis dissecans is thought to result from repetitive trauma to the weight-bearing areas and is usually seen in the lateral aspect of the medial femoral condyle. Radiographs may initially be normal, whereas in the later stages a subarticular lucency, with or without loose bodies, may be demonstrated. Signs on fat-suppressed MR images that suggest instability include: a fragment outlined by fluid; an articular fracture passing through the subchondral bone plate; a focal osteochondral defect filled with joint fluid, and a 5-mm or larger cyst deep to the lesion. The criteria above have high specificity for lesions in adults, but not in juveniles [48].

\section{Soft-tissue injuries}

Muscle contusions are frequently seen in contact sports. If the contusion becomes hard, warm and tender, development of myositis ossificans should be suspected. In this case, plain radiographs will show soft-tissue calcification that can become more extensive over time. Muscle contusions and strains appear on US as focal or diffuse swellings with increased or inhomogeneous echogenicity, and in the chronic stage as a hyperechoic atrophied scar. Haematomas may evolve into anechoic fluid-like collections. Patellar tendinopathy or 'jumper's knee' results from repetitive and forceful quadriceps contraction. On US an inhomogeneous broadening of the tendon can be seen, with marked colour Doppler hyperaemia representing neovascularization (Fig. 13). Patellar tracking disorders or a hypermobile patella are possible risk factors for patellar tendinopathy. MR imaging may directly identify signal changes along the patellar tendon insertion.

\section{Ankle-foot}

\section{Osseous injuries}

Ankle fractures in children are minimally displaced, but they may require open reduction and internal fixation if

Fig. 14 Athletic injuries in the foot and ankle. a, b A 15-yearold female athlete, practicing judo $3 \mathrm{~h}$ per day in preparation for the Olympic Games. The sagittal heavily T2-W MR image (a) shows an oblique occult fracture of the distal medial malleolus extending to the articular surface (arrow). The fatsuppressed coronal T2-W MR image (b) shows cartilaginous loose bodies within the joint effusion (arrows). c A 12-yearold basketball player with a previous ankle sprain and pain for the previous 6 months. The coronal T2-W MR image shows an unstable osteochondral fracture of the lateral talar dome (arrow). d A 14-year-old female elite athlete in technical swimming reported pain during training. The axial fat-suppressed T2-W MR image shows subcutaneous oedema in keeping with injury of the retinaculum and the extensor tendons (arrows)
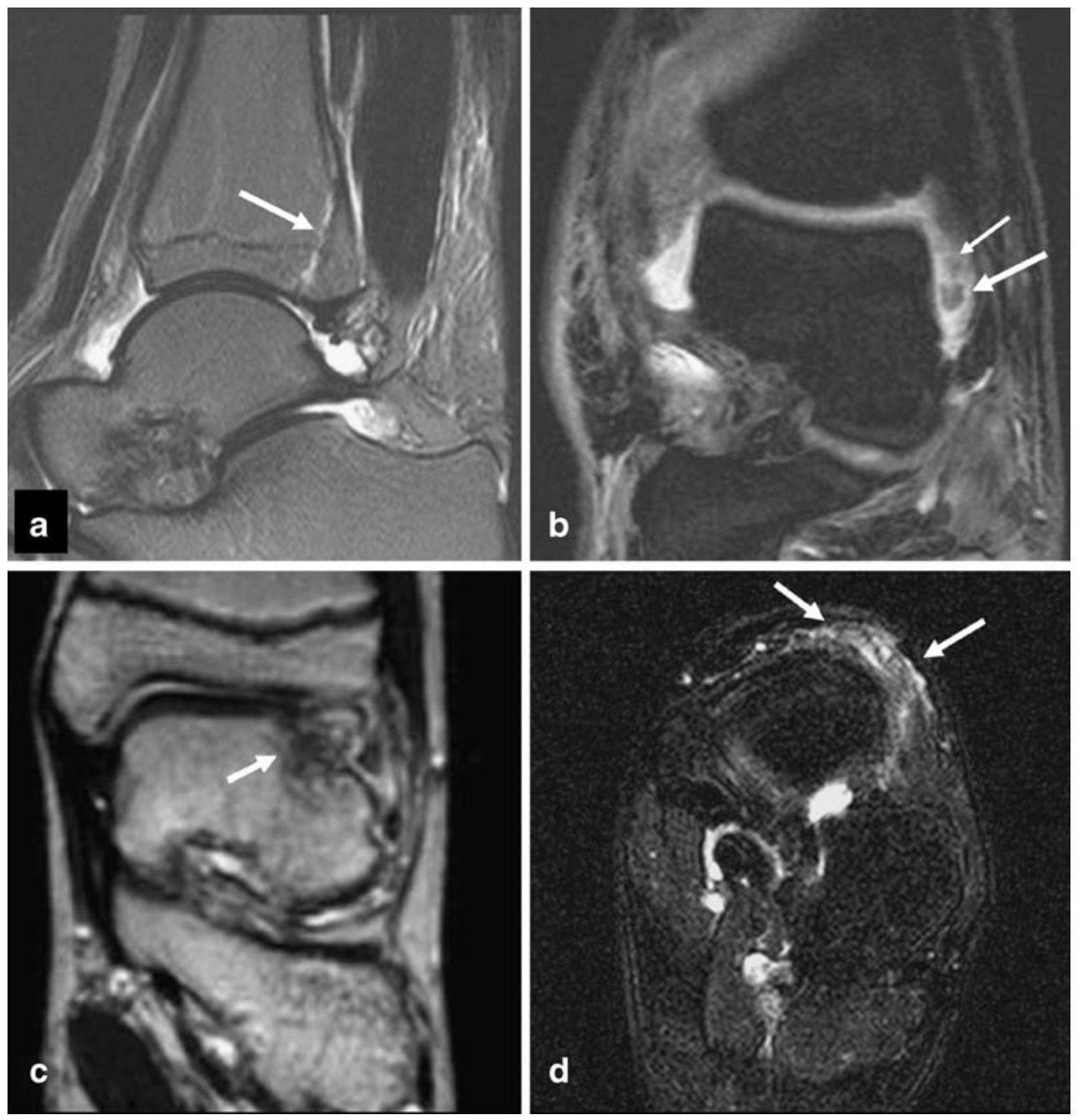
they involve the articular surface. Physeal fractures of the distal tibia and fibula may also occur. Most ankle fractures are detected radiographically. However, if the fracture is undisplaced it may be occult and will be demonstrated on MRI as a line of low signal on T1-W and high signal on T2$\mathrm{W}$ images surrounded by bone marrow oedema (Fig. 14). Bone bruises are common after single or recurrent ankle sprains and are detected only on MRI [49].

Many overuse lesions occur in the foot. Sever's lesion represents a clinical situation with posterior pain in the heel bone caused by a traction apophysitis. Since fragmentation of the calcaneal apophysis on plain radiographs is seen both in symptomatic and asymptomatic feet, imaging is not helpful. Stress fractures, mostly of the second or third metatarsals, navicular and cuboid bones and of the proximal portion of the fifth metatarsal (Iselin's disease) are also frequently encountered. The MR imaging appearance of a stress fracture is a low-signal line on T1-W and T2-W images with surrounding oedema [49].

\section{Intraarticular injuries}

Osteochondral injuries result from single or repetitive trauma. The term "osteochondral lesion" is preferred to the term "osteochondritis dissecans". These injuries in the ankle are usually located in the talar dome and should always be suspected in sprains not responding to conservative treatment. MRI is the method of choice for classifying the lesion as stable or unstable [50] (Fig. 14). In the presence of a joint effusion, cartilaginous loose bodies can be detected (Fig. 14), otherwise MR arthrography may be indicated [51].

\section{Soft tissue injuries}

Acute ligamentous injuries are rarely treated surgically and thus imaging is not usually required. MRI is reserved for when surgical repair is contemplated in elite athletes, in those with a history of chronic instability, and in those with symptoms persisting for more than 6 weeks following injury. Swimmers may show oedema surrounding the extensor tendons and the retinacula on MRI as a result of overuse and intense training (Fig. 14). Acute injuries and chronic mucinous degeneration of the Achilles tendon are rare in the growing skeleton. Subacute or chronic disorders also include the posterior impingement syndrome resulting from an os trigonum and overuse tenosynovitis [51].

\section{Conclusion}

As overuse injuries in the paediatric and adolescent population are increasing in our society and as physicians are also increasingly occupied with this type of injury, it is very important to have a better insight into the aetiology, especially the underlying risk factors and biomechanics of sport activities, as well as a knowledge of the various possibilities of imaging techniques and findings in different injuries. As the types of injury in the immature skeleton differ from those in adults due to differences in the weak points in the musculoskeletal chain, it is important for both paediatric and general radiologists to have knowledge of these differences and the resulting injuries. Although incomplete, we have, in this article, presented the most important risk factors, biomechanics, and imaging findings in overuse injuries of the upper and lower limb and spine. The ongoing evolution and availability of imaging techniques, especially MRI, has led to more possibilities to depict injuries. As misdiagnosed injuries can lead to impairment and possible irreversible damage, an increasing demand is put on clinicians and, consequently, radiologists to assess overuse injury. Imaging can be helpful not only in the primary diagnosis, but to establish indications for invasive treatment and for follow-up.

Open Access This article is distributed under the terms of the Creative Commons Attribution Noncommercial License which permits any noncommercial use, distribution, and reproduction in any medium, provided the original author(s) and source are credited.

\section{References}

1. Micheli LJ (1995) Sport injuries in children and adolescents. Questions and controversies. Clin Sports Med 14:727-745

2. Micheli LJ (1987) The traction apophysitises. Clin Sports Med 6:389-404

3. Outerbridge AR, Micheli LJ (1995) Overuse injuries in the young athlete. Clin Sports Med 14:503-516

4. Caine D, DiFiori J, Maffulli N (2006) Physeal injuries in children's and youth sports: reasons for concern. Br J Sports Med 40:749-760

5. Dalton SE (1992) Overuse injuries in adolescent athletes. Sports Med 13:58-70

6. Brenner JS (2007) Overuse injuries, overtraining, and burnout in child and adolescent athletes. Pediatrics 119:1242-1245

7. Wasserlauf BL, Paletta GA Jr (2003) Shoulder disorders in the skeletally immature throwing athlete. Orthop Clin North Am 34:427-437

8. Chen FS, Diaz VA, Loebenberg M et al (2005) Shoulder and elbow injuries in the skeletally immature athlete. J Am Acad Orthop Surg 13:172-185

9. Carson WG Jr, Gasser SI (1998) Little Leaguer's shoulder. A report of 23 cases. Am J Sports Med 26:575-580

10. Song JC, Lazarus ML, Song AP (2006) MRI findings in Little Leaguer's shoulder. Skeletal Radiol 35:107-109

11. Obembe OO, Gaskin CM, Taffoni MJ et al (2007) Little Leaguer's shoulder (proximal humeral epiphyseolysis): MRI findings in four boys. Pediatr Radiol 37:885-889

12. Popkin CA, Posada A, Clifford PD (2006) Little Leaguer's shoulder. Clin Imaging 30:365-367

13. Emery KH (2006) Imaging of sport injuries of the upper extremity in children. Clin Sports Med 25:543-568 
14. Klingele KE, Kocher MS (2002) Little league elbow: valgus overload injury in the paediatric athlete. Sports Med 32:1005-1015

15. Hang DW, Chao CM, Hang YS (2004) A clinical and roentgenographic study of Little League elbow. Am J Sports Med 32:79-84

16. Gill TJ 4th, Micheli LJ (1996) The immature athlete. Common injuries and overuse syndromes of the elbow and wrist. Clin Sports Med 15:401-423

17. DaSilva MF, Williams JS, Fadale PD et al (1998) Pediatric throwing injuries about the elbow. Am J Orthop 27:90-96

18. Sugimoto H, Ohsawa $T$ (1994) Ulnar collateral ligament in the growing elbow: MR imaging of normal development and throwing injuries. Radiology 192:417-422

19. Chang CY, Shih C, Penn IW et al (1995) Wrist injuries in adolescent gymnasts of a Chinese opera school: radiographic survey. Radiology 195:861-864

20. Shih C, Chang CY, Penn IW et al (1995) Chronically stressed wrists in adolescent gymnasts: MR imaging appearance. Radiology 195:855-859

21. Hochholzer T, Schoffl VR (2005) Epiphyseal fractures of the finger middle joints in young sport climbers. Wilderness Environ Med 16:139-142

22. Schoffl VR, Hochholzer T, Imhoff AB et al (2007) Radiographic adaptations to the stress of high-level rock climbing in junior athletes: a 5-year longitudinal study of the German Junior National Team and a group of recreational climbers. Am J Sports Med 35:86-92

23. Sward L (1992) The thoracolumbar spine in young elite athletes. Current concepts on the effects of physical training. Sports Med 13:357-364

24. Herman MJ, Pizzutillo PD (2005) Spondylolysis and spondylolisthesis in the child and adolescent: a new classification. Clin Orthop Relat Res 434:46-54

25. Campbell RS, Grainger AJ, Hide IG et al (2005) Juvenile spondylolysis: a comparative analysis of CT, SPECT and MRI. Skeletal Radiol 34:63-73

26. Bennet DL, Nassar L, DeLano MC (2006) Lumbar spine MRI in the elite-level female gymnast with low back pain. Skeletal Radiol 35:503-509

27. Sairyo K, Katoh S, Takata Y et al (2006) MRI signal changes of the pedicle as an indicator for early diagnosis of spondylolysis in children and adolescents: a clinical and biomechanical study. Spine 31:206-211

28. Hu SS, Tribus CB, Diab M et al (2008) Spondylolisthesis and spondylolysis. J Bone Joint Surg Am 90:656-671

29. Ikata T, Miyake R, Katoh S et al (1996) Pathogenesis of sportsrelated spondylolisthesis in adolescents. Radiographic and magnetic resonance imaging study. Am J Sports Med 24:94-98

30. Peh WC, Griffith JF, Yip DK et al (1998) Magnetic resonance imaging of lumbar vertebral apophyseal ring fractures. Australas Radiol 42:34-37

31. Rossi F, Dragoni S (2001) Acute avulsion fractures of the pelvis in adolescent competitive athletes: prevalence, location and sports distribution of 203 cases collected. Skeletal Radiol 30:127-131

32. Brittenden J, Robinson P (2005) Imaging of pelvic injuries in athletes. Br J Radiol 78:457-468

33. Takayanagi H, Watanabe H, Shinozaki T et al (1998) Overgrowth of the ischial tuberosity complicating femoral bone and muscle atrophy: implications for a delayed complication of malunited apophyseal avulsion fracture. Am J Orthop 27:308-312

34. Anderson SJ (2002) Lower extremity injuries in youth sports. Pediatr Clin North Am 49:627-641

35. Lee JK, Yao L (1988) Stress fractures: MR imaging. Radiology 169:217-220

36. Waters PM, Millis MB (1988) Hip and pelvic injuries in the young athlete. Clin Sports Med 7:513-526

37. Boyd KT, Peirce NS, Batt ME (1997) Common hip injuries in sport. Sports Med 24:273-288

38. Palmer WE, Kuong SJ, Elmadbouh HM (1999) MR imaging of myotendinous strain. AJR 173:703-709

39. Anderson MW, Kaplan PA, Dussault RG (2001) Adductor insertion avulsion syndrome (thigh splints): spectrum of MR imaging features. AJR 177:673-675

40. Oeppen R, Connolly S, Bencardino JT et al (2004) Acute injury of the articular cartilage and subchondral bone: a common but unrecognized lesion in the immature knee. AJR 182:111-117

41. Prince JS, Laor T, Bean JA (2005) MRI of anterior cruciate ligament injuries and associated findings in the pediatric knee: changes with skeletal maturation. AJR 185:756-762

42. Wilder RP, Sethi S (2004) Overuse injuries: tendinopathies, stress fractures, compartment syndrome, and shin splints. Clin Sports Med 23:55-81

43. Aoki Y, Yasuda K, Tohyama H et al (2004) Magnetic resonance imaging in stress fractures and shin splints. Clin Orthop Relat Res 421:260-267

44. Anderson SJ (2005) Sports injuries. Curr Probl Pediatr Adolesc Health Care 35:110-164

45. Raissaki M, Apostolaki E, Karantanas AH (2007) Imaging of sports injuries in children and adolescents. Eur J Radiol 62:86-96

46. Shea KG, Apel PJ, Pfeiffer RP (2003) Anterior cruciate ligament injury in paediatric and adolescent patients: a review of basic science and clinical research. Sports Med 33:455-471

47. Busch MT (1990) Meniscal injuries in children and adolescents. Clin Sports Med 9:661-680

48. Kijowski R, Blankenbaker DG, Shinki K et al (2008) Juvenile versus adult osteochondritis dissecans of the knee: appropriate MR imaging criteria for instability. Radiology 248:571-578

49. Sijbrandij ES, van Gils AP, de Lange EE (2002) Overuse and sports-related injuries of the ankle and hind foot: MR imaging findings. Eur J Radiol 43:45-56

50. Schachter AK, Chen AL, Reddy PD et al (2005) Osteochondral lesions of the talus. J Am Acad Orthop Surg 13:152-158

51. Rosenberg ZS, Beltran J, Bencardino JT (2000) MR imaging of the ankle and foot. Radiographics 20:S153-S179 